\title{
CONTINUA OF FINITE DEGREE AND CERTAIN PRODUCT SETS ${ }^{1}$
}

\author{
O. G. HARROLD, JR. ${ }^{2}$
}

The continua of finite degree have been studied and identified with certain well known classes of continua in a paper by G. T. Whyburn. ${ }^{3}$ The author has shown that the continua of finite degree are identical with the continua homeomorphic to a continuum of finite length. ${ }^{4}$ The object of the present note is to obtain other internal characterizations of these continua.

The symbol $M$ will represent a (compact) continuum. The continuum $M$ is said to be of finite degree at the point $p \in M$ provided that to each $\epsilon>0$ there corresponds an uncountable family of neighborhoods $(U)$ of $p$ such that (a) the diameter of each neighborhood is less than $\epsilon$, (b) each $F(U)$ is finite, where $F(U)$ is the boundary of $U$, and (c) for any pair of neighborhoods $U$ and $V$ either $\bar{U} \subset V$ or $\bar{V} \subset U$. If every point is of finite degree, the continuum $M$ is said to be of finite degree. The characterization which we find most useful below is that a continuum $M$ is of finite degree if and only if every subcontinuum contains uncountably many local separating points of $M$.

It will be shown that the classes of continua defined by each of the following properties are identical with the continua of finite degree.

Property $\mathrm{N}^{0} . M$ is locally connected and to each pair of closed, disjoint subsets $A$ and $B$ in $M$ there corresponds a finite collection of disjoint, perfect sets $H^{1}, H^{2}, \cdots, H^{k}$ such that any continuum $K$ in $M$ intersecting both $A$ and $B$ contains some $H^{i}$.

Property $Q$. If $K$ and $K_{i},(i=1,2, \cdots)$, are nondegenerate continua in $M$ with $\lim K_{i}=K$, there exists an integer $n$ such that $\prod_{n}^{\infty} K_{i}$ is an uncountable set.

It will be noted that the Property $\mathrm{N}^{0}$ is highly analogous to Property $\mathrm{N}$ which characterizes the locally connected continua such that no true cyclic element has a continuum of condensation. ${ }^{5}$

${ }^{1}$ Presented to the Society, December 29, 1939.

2 National Research Fellow.

${ }^{3} \mathrm{G}$. T. Whyburn, Concerning continua of finite degree and local separating points, American Journal of Mathematics, vol. 57, pp. 11-16.

${ }^{4}$ See abstract 45-9-321, this Bulletin.

5 This concept is due to R. L. Moore. See his Fundamental Point Set Theorems, Rice Institute Pamphlets, vol. 23, no. 1, 1936.

For this characterization see G. T. Whyburn, On continua of condensation, American Journal of Mathematics, vol. 58, pp. 705-708. 
Theorem A. A continuum is of finite degree if and only if it has Property $\mathrm{N}^{0}$.

Proof. A continuum of finite degree has the property that any pair of closed, disjoint subsets $A$ and $B$ in $M$ can be separated by a finite number of points $q^{1}, q^{2}, \cdots, q^{n}$ which are local separating points of degree two. ${ }^{3}$ Suppose $M$ is of finite degree. Set $d=\min \rho\left(q^{i}, q^{j}\right)$, $i \neq j, \rho\left(q^{i}, A+B\right)$. Since $q^{i}$ is of degree two, there exists an uncountable family of neighborhoods $\left[U\left(q^{i}\right)\right]$ such that (a) $\delta(U)<\frac{1}{2} d$, (b) $F(U)$ consists of at most two points, and (c) for any pair of distinct elements $U$ and $V$ of the family either $\bar{U} \subset V$ or $\bar{V} \subset U$. Set

$$
Y^{i}=\overline{\sum F\left(U\left(q^{i}\right)\right)},
$$

$i$ fixed. Let $H^{i}$ be a perfect subset of $Y^{i}$. There is thus determined a finite collection of disjoint, perfect sets $H^{1}, H^{2}, \cdots, H^{n}$. Let $K$ be any continuum in $M$ intersecting both $A$ and $B$. Since $M$ is of finite degree (thus hereditarily locally connected), $K$ may be taken as an arc. Since $K$ contains some $q^{i}$, it contains the corresponding $H^{i}$.

Conversely, suppose $M$ has Property $\mathrm{N}^{0}$. To prove $M$ is of finite degree it suffices to show that every nondegenerate subcontinuum in $M$ contains uncountably many local separating points of $M$. Let $K$ be a continuum in $M$ containing the distinct points $x$ and $y$. Set $A=x$, $B=y$. Then by Property $\mathrm{N}^{0}$ there exists disjoint, perfect sets $H^{1}, H^{2}, \cdots, H^{n}$ such that $K \supset H^{1}$ (say). If $K \cdot L$ is uncountable, where $L$ is the set of local separating points of $M$, our end is attained. If $K \cdot L$ is countable, there is a point $z^{1} \in H^{1}(M-L)$. Set $d=1 / 2 \min \rho\left(x, H^{i}\right), \rho\left(y, H^{i}\right), \rho\left(H^{i}, H^{j}\right), i \neq j$. Let $V$ be a region in $M$ containing $z^{1}$ which is of diameter less than $d$. Set $d^{1}=\rho\left[z^{1}, F(V)\right]$. Let $W$ be the $\frac{1}{2} d^{1}$ neighborhood of $z^{1}$, that is, the set of all points $b$ such that $\rho\left(z^{1}, b\right)<\frac{1}{2} d^{1}$. Let $X^{1}$ and $Y^{1}$ be the components of $K-K W$ containing $x$ and $y$, respectively. Since $X^{1}$ and $Y^{1}$ each contain a point of $V$ and $z^{1} \in M-L$, there exists an arc $s^{1}$ in $V-z^{1}$ joining $X^{1}$ and $Y^{1}$. Hence $K^{1}=X^{1}+s^{1}+Y^{1}$ is a continuum from $x$ to $y$ not containing $H^{1}$. If $K^{1}$ contains no other $H^{i}$, we have a contradiction to Property $\mathrm{N}^{0}$. If $K^{1} \supset H^{2}$, then $H^{2} \subset X^{1}+Y^{1}$ on account of the manner of selection of $s^{1}$. Since $K \cdot L$ is countable, $\left(X^{1}+Y^{1}\right) L$ is countable, hence there exists a point $z^{2} \in H^{2}(M-L)$. Using the same $d$ as before, a continuum $K^{2}$ is constructed (with $K^{1}$ replacing $K$ ) such that $K^{2}$ contains neither $H^{1}$ nor $H^{2}$ and $K^{2}$ intersects both $A$ and $B$. Performing these steps (with $z^{2}$ replacing $z^{1}$ ) will clearly give a continuum $K^{2}=X^{2}+s^{2}+Y^{2}$ not containing $H^{2}$, and since $s^{2} H^{1}=0$ and $X^{2}+Y^{2} C K^{1}$, we have $K^{2}$ contains neither $H^{1}$ nor $H^{2}$. After a finite number of such steps a con- 
tinuum $K^{i}$ is obtained which intersects both $A$ and $B$ and contains no $H^{i}$. This completes the proof that having Property $\mathrm{N}^{0}$ is equivalent to being of finite degree.

Theorem B. A continuum is of finite degree if and only if it has Property Q.

Proof. Let $M$ be of finite degree. Since this implies that $M$ is hereditarily locally connected, it may be assumed without loss of generality that the continua $K_{i}$ such that $\lim K_{i}=K$ are arcs. Let the end-points of $K_{i}$ be $a_{i}$ and $b_{i}$. Suppose $\lim a_{i}=x$ and $\lim b_{i}=y$. Since $K$ by assumption is a nondegenerate continuum, we may take $x \neq y$. In a continuum of finite degree the points of degree two are dense on every subcontinuum, hence let $z$ be a point of degree two in $K-(x+y)$. Let $X, Y$ and $Z$ be neighborhoods of $x, y$ and $z$ respectively, such that $(\bar{X} \cdot \bar{Z}+\bar{X} \cdot \bar{Y}+\bar{Y} \cdot \bar{Z})=0$. To $Z$ there corresponds an uncountable family of neighborhoods $(U)$ such that $\bar{U} \subset Z$ and $F(U)$ consists of at most two points. There is a definite $V=V(z)$ such that $\bar{V} \subset U$ for uncountably many $U$. Since $z \in \lim K_{i}$, there is an integer $n$ such that $\operatorname{arcs} K_{i}, i \geqq n$, intersect $V$, hence if we take $m \geqq n$ so large that $a_{i} \in X$, $b_{i} \in Y$ for $i \geqq m$, then $K_{i}$ must contain two boundary points of each $U$ for which $V \subset U \subset Z$. Thus $\prod_{m}^{\infty} K_{i}$ is uncountable.

To show that Property $Q$ implies finite degree we show that infinite degree implies Property non- $Q$. It is clear that a continuum containing a convergence continuum has Property non- $Q$, hence we need consider only hereditarily locally connected continua. Let $T$ be an arc in the continuum $M$ which contains only a countable number of the local separating points of $M$. For each positive integer $n$ let $W_{n}$ be the set of all points of $M$ at a distance not greater than $1 / n$ from $T$. Let $M_{n}$ be the component of $W_{n}$ which contains $T$. Let $L_{n}$ be the set of all points which separate the end-points $a$ and $b$ of $T$ in $M_{n}$. Then in $M_{n}$ there exist arcs $s_{n}$ and $t_{n}$ from $a$ to $b$ such that ${ }^{6} s_{n} t_{n}=a+L_{n}+b$. Set $K_{2 n-1}=s_{n}$ and $K_{2 n}=t_{n}$. Then $\lim K_{n}=T$. But $\prod_{n}^{\infty} K_{i}$ is at most countable, no matter what positive integer $n$ is. For $K_{2 n-1}$ and $K_{2 n}$ have only a countable number of common points, namely, $a+L_{n}+b$. Hence $M$ does not have Property $Q$.

The University of Virginia

${ }^{6}$ G. T. Whyburn, Some properties of continuous curves, this Bulletin, vol. 33 (1927), pp. 305-308. See Theorcm 3. 